\title{
Accrual Status Date
}

National Cancer Institute

\section{Source}

National Cancer Institute. Accrual Status Date. NCI Thesaurus. Code C93494.

The date (and time) on which the accrual status is established. 